\title{
Reactive Gas Ion Beam Generation Using Single Atom W(111) Gas Field Ion Sources
}

\author{
R. $\operatorname{Urban}^{1,2}$, H. Moritani ${ }^{2,3}$, R.A. Wolkow ${ }^{1,2}$ and J.L. Pitters ${ }^{2}$ \\ 1. Department of Physics, University of Alberta, Edmonton, AB, Canada \\ 2. National Institutes for Nanotechnology, Edmonton, AB, Canada \\ 3. Hitachi High-Tech Science Corporation, Shizuoka, Japan
}

The scanning ion microscopy is gaining momentum as it provides several key advantages over scanning electron microscopy: (i) enhanced depth of focus, (ii) improved surface and element sensitivity, (iii) better lateral resolution, and (iv) nanomachining and milling. It uses different ions to achieve these tasks ranging from inert gases like helium and neon for imaging and ion milling. Other gases such as argon, nitrogen, and oxygen have potential for further sputtering and etching. It is therefore crucial that gas field ion sources provide necessary robustness and stability for range of various gases.

It has been demonstrated that iridium SATs can be used to generate both nitrogen and oxygen beams [1]. This is not surprising since iridium is a noble metal of a platinum group. It is also considered as one of the most corrosive-resistant metal at temperatures as high as $2000 \mathrm{~K}$. However, GFISs operate at high electric fields and thus do not obey conventional corrosion rules. It has been demonstrated that both iridium and platinum can be etched and shaped by the nitrogen- and oxygen-assisted etching and evaporation process in the same manner as tungsten [2]. Yet, it provides suitable base for nitrogen and oxygen beam generation. It raises an interesting question: Can W(111) SAT be used for nitrogen beam generation? The answer lies in understating of nitrogen-assisted etching (nitrogen corrosion) and nitrogen beam generation.

The nitrogen etching process is described in several steps: (i) nitrogen molecules cover the shank of a tip and migrate towards the apex in presence of electric field. (ii) Simultaneously, nitrogen affects underlying tungsten lattice creating atomic protrusions where electric field is locally enhanced over the unaffected surface. (iii) If these protrusions happen in the low field region, nothing happens. If the field is strong enough, field evaporation of $\mathrm{W}$ atom occurs resulting in etching.

We have prepared SATs using a combination of etching and annealing in nitrogen atmosphere (980C, 5 minutes, $5 \times 10^{-5}$ torr of $\mathrm{N}_{2}$ ). The tip voltage is gradually increased to reach best imaging fields for nitrogen, oxygen, and hydrogen, respectively, see Figure 1. The beams appeared to be as stable as helium one under similar conditions. It should be noted that the best imaging fields for nitrogen (17 $\mathrm{V} / \mathrm{nm})$, hydrogen $(22 \mathrm{~V} / \mathrm{nm})$, and oxygen $(15 \mathrm{~V} / \mathrm{nm})$ are significantly lower than those of helium (44 $\mathrm{V} / \mathrm{nm})$ and neon $(35 \mathrm{~V} / \mathrm{nm})[3]$.

The reason for the SAT stability and resilience to nitrogen etching comes from the fact that strong electric field is required for etching to occur. It is clear that nitrogen-assisted etching relies on electric field to field evaporate affected tungsten atoms. In the absence of field there would be no corrosion. Similarly, if the field is too strong (nitrogen cannot adsorb) or too weak (no field evaporations), no corrosion occurs. It follows that BIF for nitrogen ionization is significantly lower than field required to field evaporate "reacted" tungsten atom. 
In conclusion, gas field ion sources based on W(111) SATs can be used to generate nitrogen, oxygen, and hydrogen ion beams despite its reactivity and etching properties. Stability and robustness of W(111) apex against nitrogen/oxygen etching during ion beam generation is due to low nitrogen/oxygen ionization field which inhibits tungsten etching. This is an important conclusion allowing W(111) SATs to serve as universal GFIS for scanning ion microscopy.

References:

[1] H.-S. Kuo et al, Nanotechnology 20, 335701 (2009)

[2] J. Wood et al, Iridium single atom tips by field assisted reactive gas etching, accepted to Appl. Surf. Sci. (2015)

[3] E.W. Muller, T.T. Tsong, Field Ion Microscopy, Elsevier, New York (1969)
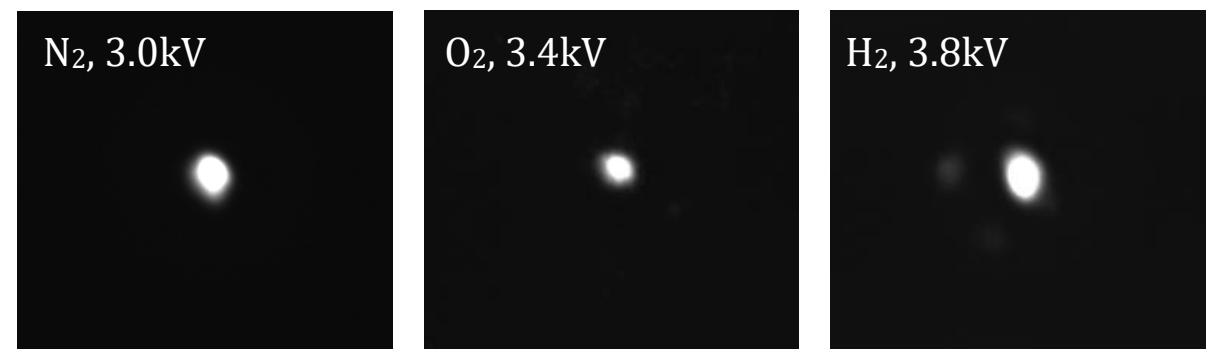

Figure 1. A typical W(111) SAT prepared by annealing in presence of nitrogen. Stable ion beams were generated using (a) nitrogen, (b) oxygen, and (c) hydrogen. 